\title{
Sensitivity Enhancement by Carbon Nanotubes: Applications to Stem Cell Cultures Monitoring
}

\author{
Cristina Boero, Sandro Carrara and Giovanni De Micheli \\ Ecole Polytechnique Fédérale de Lausanne \\ $\mathrm{CH}$ - 1015 Lausanne $(\mathrm{CH})$ \\ Email: cristina.boero@epfl.ch
}

\begin{abstract}
Nano-biosensing provides new tools to investigate cellular differentiation and proliferation. Upon the various metabolic compounds secreted by cells during life cycles, glucose, lactate and hydrogen peroxide $\left(\mathrm{H}_{2} \mathrm{O}_{2}\right)$ are of first interest. Nanostructured electrodes may enhance the compounds sensitivity in order to precisely detect cell cycle variation. In the present paper, the detection with electrodes nanostructured by using MultiWalled Carbon Nanotubes (MWCNT) was investigated in order to develop an amperometric biosensor. Good improvement in sensitivity was obtained, suggesting that carbon nanotubes can be the right candidates to improve biosensing. The final aim of the study is the development of a bio-chip, which can be integrated in Petri dishes for automatic stem cell culture monitoring.
\end{abstract}

\section{INTRODUCTION}

Despite the worldwide expansion of biotechnology, automatic monitoring of cell bioprocesses is still rather underdeveloped. There is a lot of literature about development of biosensors for clinical purposes and surely the most popular application is for glucose monitoring in diabetes pathology. However, not so many devices are available for real-time analysis of metabolic compounds in cell culture medium. A clear identification of medium compounds, which may be identified as markers of different stem cell states enabling identification in specialized tissue, is still not available. Monitoring such parameters as glucose, lactate and $\mathrm{H}_{2} \mathrm{O}_{2}$ can give important information about how the culture is evolving and growing, especially when it is applied to stem cell culture [1]. In particular, glucose marks the cell energetic demand, while lactate and hydrogen peroxide mark the cell suffering. Electrochemical enzyme sensors can be of much interest, particularly those based on amperometry. They can pave the way to automated systems, saving time to human work and representing a further instrument to investigate cellular differentiation and proliferation.

To date, only few process variables are commonly monitored, e.g. $\mathrm{pH}, \mathrm{pO}_{2}$, electronic impedance and temperature [2]. The final goal of the research is developing Petri dishes equipped with biosensors for the detection of metabolic compounds released into the cell medium during cell differentiation with improved characteristics respect to the present state-of-the-art in cell culture monitoring. The first stage of the complete research is here summarized and focused on the optimization of the electrodes employing nanostructures like MultiWalled Carbon Nanotubes, in order to tune a sensor for the detection of $\mathrm{H}_{2} \mathrm{O}_{2}$. We started with pure hydrogen peroxide

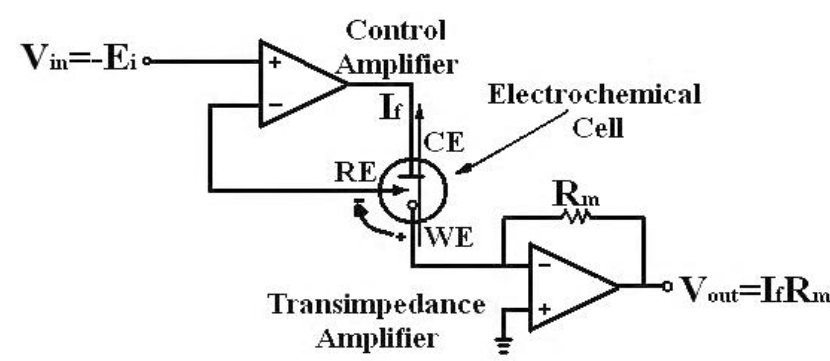

Fig. 1. Schematic of a simplified potentiostat with the potential control and the amperometric measurement configurations. Reprinted from [4].

detection, also because LOD and GOD produce it during enzymatic reaction. Moreover, hydrogen peroxide is one of the major oxidative stimuli in antioxidative studies and it is a symptom of cell sufferance. Previous studies [3] showed that carbon nanotubes can impart strong electrocatalytic activity in electrochemical devices, owning the ability to promote the electron-transfer from the substrate to the electrode surface in the presence of a redox reaction. Cyclic voltammetry and chronoamperometry will be used to investigate the biosensor behavior and to characterize it in term of selectivity and sensitivity.

\section{DEVICE OVERVIEW}

After a first phase of electrode optimization for $\mathrm{H}_{2} \mathrm{O}_{2}$ detection (shown in this paper) and electrode functionalization with oxidase, e.g. lactate oxidase (LOD) and glucose oxidase (GOD), it will necessary to design the electronics for the biochip. A potentiostat is required to apply a constant potential between the working and reference electrodes and to read the current flowing between the working and counter electrodes. Mainly these tasks can be realized by means of two blocks, one for the potential control and one for the current measurement. To this end, there are a few configurations presented in literature for both of the blocks. One possible configuration for a potentiostat is shown in Fig. 1 [4]. The block on the left is for the potential configuration: the working electrode is kept at the ground potential and an operational amplifier, called the control amplifier, controls the cell current, $\mathrm{I}_{F}$, such that the cell potential, $\mathrm{V}_{\text {cell }}$, is kept at the desired preset potential, $\mathrm{E}_{i}$. The block on the right shows a possible architecture for current measurement as a simple transimpedance amplifier, 


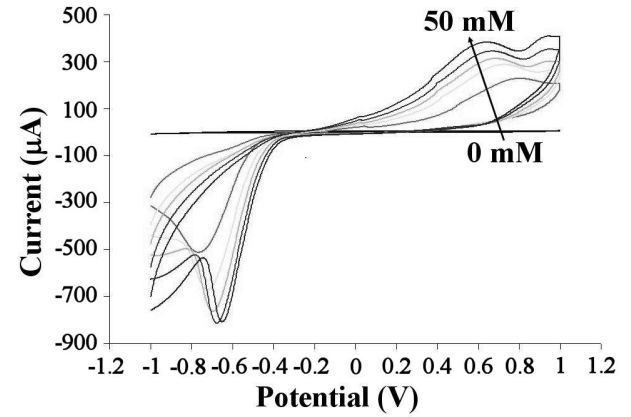

Fig. 2. Cyclic voltammetry of SPE for concentration of $\mathrm{H}_{2} \mathrm{O}_{2}$ in Milli-Q from 0 to $50 \mathrm{mM}$ by step of $10 \mathrm{mM}$. Scan rate $100 \mathrm{mV} \mathrm{s}^{-1}$.

which generates an output voltage linearly proportional to $\mathrm{I}_{F}$. Moreover, the working electrode is forced at virtual ground, so potential and current can be measured with reference to ground [4]. This solution can be easily implemented in a CMOS technology. Last step will be the validation of the biochip in real culture cell medium (neural and stem cell lines).

\section{MATERIALS AND METHODS}

\section{A. Materials}

Carbon paste screen printed electrodes (SPE) were purchased from Dropsens (model DRP-110). The electrodes were made of a graphite working electrode (area equal to about 13 $\mathrm{mm}^{2}$ ), a graphite counter electrode and an $\mathrm{Ag} / \mathrm{AgCl}$ reference electrode. The total area of the cell is $22 \mathrm{~mm}^{2} . \mathrm{H}_{2} \mathrm{O}_{2}$ (weight $30 \%$ ) was purchased from Reactolab SA (Switzerland) and it was diluted in phosphate buffer solution (PBS) $0.1 \mathrm{M}$ at $\mathrm{pH}$ 7.4. Multi-walled carbon nanotubes (diameter $10 \mathrm{~nm}$, length 1$2 \mu \mathrm{m})$ were purchased in powder (90\% purity) from Dropsens (Spain), diluted in chloroform to the concentration of $1 \mathrm{mg}$ $\mathrm{ml}^{-1}$ [3]. Samples were then sonicated in order to obtain an homogeneous solution.

\section{B. Preparation of electrodes}

The working electrode was prepared covering the SPE with a total of $60 \mu \mathrm{l}$ of MWCNT solution adding $5 \mu \mathrm{l}$ each step and it was allowed to dry. They were stored at room temperature when not used.

\section{Electrochemical measurements}

The electrochemical response of electrodes was investigated by cyclic voltammetry and chronoamperometry under aerobic conditions. Voltammograms were acquired by using a Versastat 3 potentiostat (Princeton Applied Technologies). The electrodes were covered with $100 \mu \mathrm{l}$ of PBS $0.1 \mathrm{M}$ at pH 7.4, in which was added $1 \mu \mathrm{l}$ of hydrogen peroxide sample at a time. The potential was swept in the range from -1 to $+1 \mathrm{~V}$ vs $\mathrm{Ag} / \mathrm{AgCl}$, using a scan rate of $100 \mathrm{mV} \mathrm{s}^{-1}$.

\section{RESULTS AND DISCUSSION}

\section{A. Cyclic Voltammetry $(\mathrm{CV})$}

Since oxidases produce hydrogen peroxide in the enzymatic reaction, we investigate its electrochemical behavior

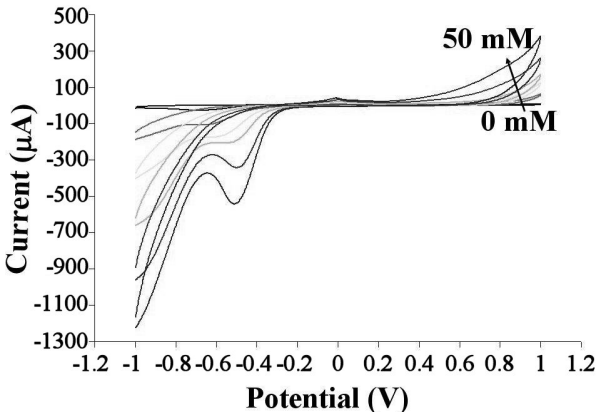

Fig. 3. Cyclic voltammetry of SPE for concentration of $\mathrm{H}_{2} \mathrm{O}_{2}$ in PBS from 0 to $50 \mathrm{mM}$ by step of $10 \mathrm{mM}$. Scan rate $100 \mathrm{mV} \mathrm{s}^{-1}$.

with cyclic voltammetry and chronoamperometry. In fact, for glucose and lactate, the enzymatic reaction is the following $(\mathrm{X}=\mathrm{L}$ or $\mathrm{G})$ :

$$
\begin{gathered}
X O D / F A D+\text { substrate } \rightarrow X O D / F A D H_{2}+\text { product } \\
X O D / \mathrm{FADH}_{2}+\mathrm{O}_{2} \rightarrow \mathrm{H}_{2} \mathrm{O}_{2}+\mathrm{XOD} / \mathrm{FAD}
\end{gathered}
$$

where pyruvate and gluconic acid are enzymatic reaction products for lactate and glucose, respectively. So, from the biosensing point-of-view, it is interesting to investigate hydrogen peroxide and its electrochemical characteristics. We started with $\mathrm{CV}$, in order to identify the related oxidation peak and the potential at which chronoamperometries should be carried out to detect compounds of interest. Cyclic voltammetries of $\mathrm{H}_{2} \mathrm{O}_{2}$ were performed using Milli-Q as support electrolyte, to avoid possible interferences due to ions contained in PBS. Fig. 2 shows the voltammograms related to addition of 10 $\mathrm{mM}$ of hydrogen peroxide each step. The range is from 0 up to $50 \mathrm{mM}$, because a high concentrations is required to detect the compound in non-structured electrodes. It can be observed from the graph that there is a big reduction peak around $-700 \mathrm{mV}$, which correspond to the first term of Eq. (2) and it is about the value reported in literature for the potential reduction of $\mathrm{O}_{2}$ [5]. Therefore, if that potential value is applied in the enzymatic reaction, the measurement of decreasing oxygen concentration is detected. This kind of electrode is called Clark oxygen electrode, but it has some correlated problems. First of all, the ambient level of oxygen needs to be controlled and constant, otherwise the electrode response to the decrease in oxygen concentration would not be proportional to the decrease in substrate concentration. Another problem is the high reduction potential needed to reduce oxygen, which other compounds might interfere with. Another peak is visible around $+650 \mathrm{mV}$ and it is associated to $\mathrm{H}_{2} \mathrm{O}_{2}$ oxidation, as reported in the second term of Eq. (2) and in literature [5]. This is the peak of interest for the present application. The reaction is the following:

$$
2 \mathrm{H}_{2} \mathrm{O}_{2} \rightarrow 2 \mathrm{H}_{2} \mathrm{O}+\mathrm{O}_{2}^{+}+4 e^{-}
$$

and the four $\mathrm{e}^{-}$released to the electrodes are optimally detected at $+650 \mathrm{mV}$. Cyclic voltammetries were also carried out in PBS as support electrolyte, because it is closer to 


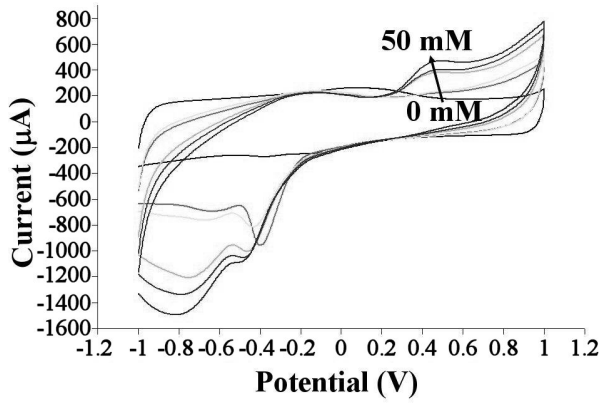

Fig. 4. Cyclic voltammetry of nanostructured SPE for concentration of $\mathrm{H}_{2} \mathrm{O}_{2}$ in PBS from 0 to $50 \mathrm{mM}$ by step of $10 \mathrm{mM}$. Scan rate $100 \mathrm{mV} \mathrm{s}^{-1}$.

biological fluids (see Fig. 3). The peak of $\mathrm{O}_{2}$ reduction is still present at a potential of around $-500 \mathrm{mV}$, but we cannot observe the oxidation peak related to $\mathrm{H}_{2} \mathrm{O}_{2}$. It means we need higher concentration range, because it is not possible to identify the oxidation peak and the reduction peak is smaller than in the previous case. Thus, the PBS as supporting electrolyte is partially hiding the $\mathrm{H}_{2} \mathrm{O}_{2}$ detection. In the case of nanostructured electrodes with MWCNT (see Fig. 4) the two peaks appear again. The oxidation peak is the most interesting. It is visible only from $30 \mathrm{mM}$, even if it can be observed a current increase adding $\mathrm{H}_{2} \mathrm{O}_{2}$ for less amount. The second important result is that oxidation potential is lower respect to the previous case: in fact, here the peak is around $+500 \mathrm{mV}$. It is a behavior that was already observed in previous works [3]. Similarly, also the $\mathrm{O}_{2}$ reduction peak shifts toward lower absolute potentials. Peak potential is a function of substrate concentration according to Nernst equation:

$$
E=E^{0}+\frac{R T}{n F} \ln \left(\frac{C_{o x}}{C_{r e d}}\right)
$$

where $E^{0}$ is the standard electrode potential, $R$ is the molar gas constant, $T$ is the absolute temperature, $n$ is the number of electrons transferred in the reaction, $F$ is the Faraday's constant, $C_{o x}$ and $C_{r e d}$ are the concentration of the oxidized and reduced forms of the species, respectively. Since from Nernst equation it is not clear the parameters that can change adding carbon nanotubes onto the electrode, the hypothesis is that CNT could modify the electrostatic attraction in the double layer that is formed onto the interface between the electrode and the solution. That hypothesis was directly correlated with biosensing [6] and it can be a possible explanation of very promising properties belonged to MWCNT [7]. This layer effect changes the target molecule concentration close to electrode and concentration ratio changes in Nernst equation. This is a great advantage: direct amperometric detection of $\mathrm{H}_{2} \mathrm{O}_{2}$ requires a higher potential at an unmodified electrode which causes severe interference arising from easily electrooxidizable substances such as ascorbic and uric acid existing inherently in biological fluids. Consequently, it is possible to overcome the influence due to other compounds by reducing the applied potential with the use of casted carbon nanotubes.

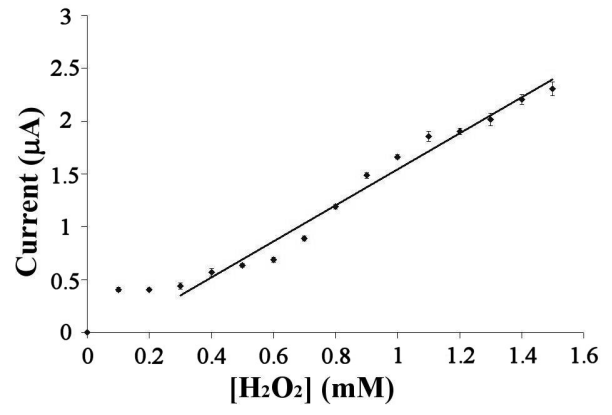

Fig. 5. Calibration curves obtained from chronoamperometry for the range from 0 to $1.5 \mathrm{mM}$ of $\mathrm{H}_{2} \mathrm{O}_{2}$ in PBS by step of $0.1 \mathrm{mM}$ for SPE with MWCNT.

\section{B. Chronoamperometry}

Preliminary results were carried out with chronoamperometry in two concentration ranges for hydrogen peroxide. From this technique it is possible to obtain the sensitivity of the biosensor at given potential. According to the results obtained from CV, a chronoamperometry on non-structured SPE was performed at $+650 \mathrm{mV}$ in the range of $0-1500 \mu \mathrm{M}$ of $\mathrm{H}_{2} \mathrm{O}_{2}$. This range was chosen because it is closer to the hydrogen peroxide concentration in cell culture ([8]) and it is also in the range for lactate detection, which allows a comparison with other authors. Stoichiometric rate between lactate and hydrogen peroxide is $1: 1$, so it is possible performing a comparison with sensitivity values obtained with lactate biosensors. The response time of the system after every addition of lactate was about $40 \mathrm{~s}$; after that time, another drop of substrate was casted. The calibration curve related to bare electrode is not shown, because the current increase due to following additions of $\mathrm{H}_{2} \mathrm{O}_{2}$ was too small. Instead, nanostructured electrodes show better response. As it can see from Fig. 5, the system seems to be insensitive to hydrogen peroxide up to $300 \mu \mathrm{M}$. The calibration curve reported into the figure correspond to a sensitivity of $12.8 \mu \mathrm{A} \mathrm{mM}^{-1} \mathrm{~cm}^{-2}$. Table I summarizes the sensitivity values from previous works. It could be observed that biosensors which don't use carbon nanotubes have a sensitivity of magnitude less, even if they are using polymer matrixes. These results show great promises for the use of carbon nanotubes in biosensing. The sensitivity obtained by the present study is a little bit smaller compared to that one showed by Cui et al. [9], but it can be explained by the fact that they use oxidase in polymeric matrix, which can enhance the efficiency, despite of the cost and complexity of the sensor. To investigate the sensitivity gain obtained by nanostructuring, chronoamperometries were performed in the range of $0-12 \mathrm{mM}$. This is the useful range for lactate and glucose biosensors in cell monitoring. The concentration range for cell culture is from 0 to $12.5 \mathrm{mM}$ for lactate and from 0 to $6 \mathrm{mM}$ for glucose [10]. The calibration curve is plotted in Fig. 6 . It could be noticed a great improvement in sensitivity: from $0.79 \mu \mathrm{A} \mathrm{mM}^{-1} \mathrm{~cm}^{-2}$ in the case of non-structured SPE up to $5.8 \mu \mathrm{A} \mathrm{mM}^{-1} \mathrm{~cm}^{-2}$ in the case of nanostructured electrodes; it means that sensitivity in the case of carbon nanotubes is 7 


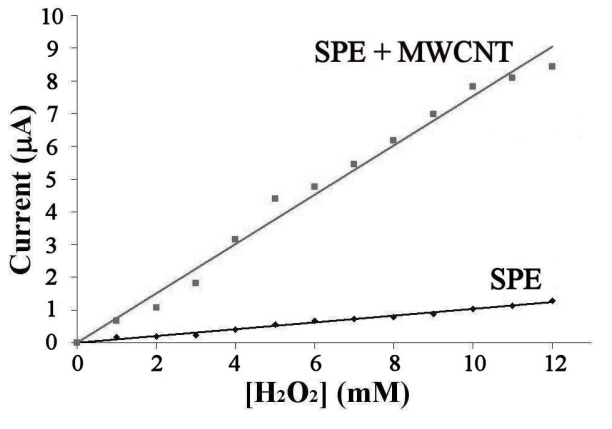

Fig. 6. Calibration curves obtained from chronoamperometry data analysis for the range from 0 to $12 \mathrm{mM}$ of $\mathrm{H}_{2} \mathrm{O}_{2}$ with step of $1 \mathrm{mM}$ in the case of SPE and SPE functionalized with MWCNT.

times higher than without them. This is an interesting result and it confirms another time that carbon nanotubes enhance signals coming from sensors.

\section{CONCLUSION}

This paper presents preliminary results in the optimization of electrodes for metabolites detection in cell culture medium. Lactate, glucose, glutamate and other compounds can be detected by meaning of oxidases, which produce hydrogen peroxide. Data from electrochemical acquisition on carbon based screen printed electrodes have been shown. The electrodes were nanostructured by using Multi-Walled Carbon Nanotubes.

Cyclic voltammetry was performed in different experimental conditions (PBS or Milli-Q as support electrolyte) with different hydrogen peroxide concentrations. In the case of Milli$\mathrm{Q}$, two peaks can be clearly identified: the peak at positive potential $(+650 \mathrm{mV})$ is related to hydrogen peroxide oxidation, while that at negative potential is related to oxygen reduction $(-700 \mathrm{mV})$. Also in PBS these two peaks appear, but for higher peroxide concentration. For all cases current increases proportionally with concentration, but it is much more evident in case of nanostructured electrodes. Moreover, oxidation peak appears at a lower potential in the case of electrode structured by using MWCNT.

In the range of $0-1.5 \mathrm{mM}$, the sensitivity for SPE functionalized with MWCNT corresponds to $12.8 \mu \mathrm{A} \mathrm{mM} \mathrm{mM}^{-1} \mathrm{~cm}^{-2}$, which is higher than almost all the values found in literature. In the range of $0-12 \mathrm{mM}$ the sensitivity value is $5.8 \mu \mathrm{A} \mathrm{mM}^{-1}$ $\mathrm{cm}^{-2}$ for electrodes with MWCNT, 7 times higher than for SPE without MWCNT. All this results confirm the attitude of carbon nanotubes to promote the electron-transfer from the substrate onto the surface electrode and confirm that hydrogen peroxide electrochemical detection may be improved by the use of MWCNT. Future work will focus on microfabrication of nanostructured electrodes and tuning for lactate and glucose detection, design and testing of the PCB for current detection and RF transmission, fabrication and validation of the VLSI bio-chip.
TABLE I

SENSITIVITY VALUES FROM LITERATURE

\begin{tabular}{c|c}
\hline Methods & Sensitivity \\
\hline \hline Au-Nafion@- TNTs [11] & $0.24 \mu \mathrm{A} \mathrm{mM}^{-1} \mathrm{~cm}^{-2}$ \\
\hline Polypyrrole - polyanion/PEG [12] & $0.15 \mu \mathrm{A} \mathrm{mM}^{-1} \mathrm{~cm}^{-2}$ \\
\hline MWCNT-chitosan [13] & $8.3 \mu \mathrm{A} \mathrm{mM}^{-1} \mathrm{~cm}^{-2}$ \\
\hline chitosan/PVI-Os/CNT [9] & $19.7 \mu \mathrm{A} \mathrm{mM}^{-1} \mathrm{~cm}^{-2}$ \\
\hline casted MWCNT (Present study) & $12.8 \mu \mathrm{A} \mathrm{m}^{-1} \mathrm{~cm}^{-2}$ \\
\hline
\end{tabular}

\section{ACKNOWLEDGMENT}

The authors would like to thank L. Calzà and G. Del Vecchio for discussions on cell monitoring and D. Albini for discussions on Nernst equation related to MWCNT role. The research is financed by SNF Sino-Swiss cooperation project n. IZLCZ2 123967 and by EPFL - Integrated Center SI.

\section{REFERENCES}

[1] V. Vojinovic, F.M.F. Esteves, J.M.S. Cabral, and L.P. Fonseca. Bienzymatic analytical microreactors for glucose, lactate, ethanol, galactose and 1-amino acid monitoring in cell culture media. Analytica Chimica Acta, 565(2):240 - 249, 2006.

[2] J. Wiest, M. Schmidhuber, J. Ressler, A. Scholz, M. Brischwein, and B. Wolf. Cell based assays for diagnostic and therapy on multiparametric biosensor chips with an intelligent mobile lab. IFMBE Proc., 10:132135, 2005.

[3] S. Carrara, V. Shumyantseva, A.I. Archakov, and B. Samor. Screenprinted electrodes based on carbon nanotubes and cytochrome p450scc for highly sensitive cholesterol biosensors. Biosensors and Bioelectronics, 24(1): 148 - 150, 2008 .

[4] K. Iniewski. VLSI Circuits for Biomedical Applications. Artech House, 2008. pp. 265-285.

[5] B. R. Eggins. Chemical sensors and biosensors. John Wiley \& sons. pp. $125-169$.

[6] S. Carrara, V. Bavastrello, D. Ricci, E. Stura, and C. Nicolini. Improved nanocomposite materials for biosensor applications investigated by electrochemical impedance spectroscopy. Sensors and Actuators B: Chemical, 109(2):221 - 226, 2005.

[7] H. Pan, C.K. Poh, Y.P. Feng, and J. Lin. Supercapacitor electrodes from tubes-in-tube carbon nanostructures. Chemistry of Materials, 19(25):6120-6125, 2007.

[8] G.F. Shi, L.J. An, B. Jiang, S. Guan, and Y.M. Bao. Alpinia protocatechuic acid protects against oxidative damage in vitro and reduces oxidative stress in vivo. Neuroscience Letters, 403(3):206 - 210, 2006.

[9] X. Cui, C. M. Li, J. Zang, and S. Yu. Highly sensitive lactate biosensor by engineering chitosan/pvi-os/cnt/lod network nanocomposite. Biosensors and Bioelectronics, 22(12):3288 - 3292, 2007.

[10] Y.S. Hwang, J. Cho, F. Tay, J.Y.Y. Heng, R. Ho, S.G. Kazarian, D.R. Williams, A.R. Boccaccini, J.M. Polak, and A. Mantalaris. The use of murine embryonic stem cells, alginate encapsulation, and rotary microgravity bioreactor in bone tissue engineering. Biomaterials, 30(4):499 $-507,2009$.

[11] M. Yang, J. Wang, H. Li, J.G. Zheng, and N.N. Wu. A lactate electrochemical biosensor with a titanate nanotube as direct electron transfer promoter. Nanotechnology, 19(7):075502 (6pp), 2008.

[12] W.J. Sung and Y.H. Bae. Glucose oxidase, lactate oxidase, and galactose oxidase enzyme electrode based on polypyrrole with polyanion/peg/enzyme conjugate dopant. Sensors and Actuators B: Chemical, 114(1): 164 - 169, 2006.

[13] Y.C. Tsai, S.Y. Chen, and H.W. Liaw. Immobilization of lactate dehydrogenase within multiwalled carbon nanotube-chitosan nanocomposite for application to lactate biosensors. Sensors and Actuators B: Chemical, 125(2):474 - 481, 2007. 